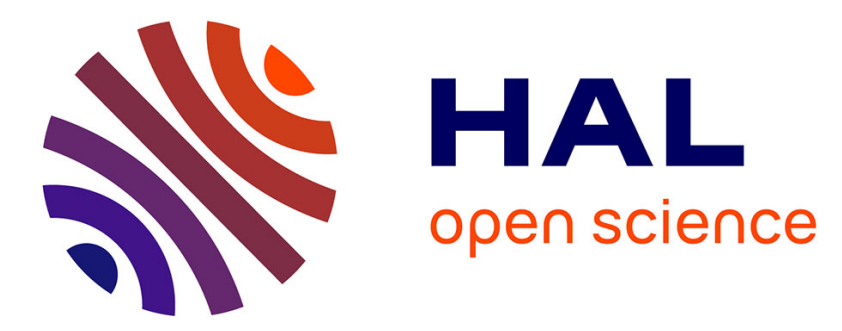

\title{
Drastic bandwidth enhancement of bistable energy harvesters: study of subharmonic behaviors and their stability robustness
}

Thomas Huguet, Adrien Badel, Olivier Druet, Mickael Lallart

\section{- To cite this version:}

Thomas Huguet, Adrien Badel, Olivier Druet, Mickael Lallart. Drastic bandwidth enhancement of bistable energy harvesters: study of subharmonic behaviors and their stability robustness. Applied Energy, 2018, 226, pp.607 - 617. 10.1016/j.apenergy.2018.06.011 . hal-01901836v2

\section{HAL Id: hal-01901836 \\ https://hal.science/hal-01901836v2}

Submitted on 29 Apr 2019

HAL is a multi-disciplinary open access archive for the deposit and dissemination of scientific research documents, whether they are published or not. The documents may come from teaching and research institutions in France or abroad, or from public or private research centers.
L'archive ouverte pluridisciplinaire HAL, est destinée au dépôt et à la diffusion de documents scientifiques de niveau recherche, publiés ou non, émanant des établissements d'enseignement et de recherche français ou étrangers, des laboratoires publics ou privés. 


\title{
Drastic bandwidth enhancement of bistable energy harvesters: study of subharmonic behaviors and their stability robustness
}

\author{
Thomas Huguet ${ }^{\mathrm{a}, \mathrm{b}}$, Adrien Badel ${ }^{\mathrm{b}}$ Olivier Druet ${ }^{\mathrm{c}}$ and Mickaël Lallart ${ }^{\mathrm{a},{ }^{*}}$ \\ a Univ. Lyon, INSA-Lyon, LGEF, EA682, F-69621, Villeurbanne, France \\ b Univ. Savoie Mont-Blanc, SYMME, F-74000, Annecy, France \\ c Univ. Lyon, Institut Camille Jordan, F-69621, Villeurbanne, France \\ * corresponding author: mickael.lallart@insa-lyon.fr
}

\begin{abstract}
In order to provide a serious alternative to chemical batteries for the energy supply of isolated sensors, bistable generators have been enthusiastically highlighted in recent years for their ability to harvest vibration energy on a wider frequency range compared to linear generators. Nevertheless, these bistable harvesters are generally characterized through a frequency sweep which does not reveal all the steady-state behaviors they can reach and therefore their full energy harvesting potential. Among such behaviors, subharmonic motions are hidden by this classical characterization and therefore had not received a lot of attention. This study proposes an original complete analytical analysis of subharmonic orbits for energy harvesting to predict their contribution to the global bandwidth of bistable generators. In addition, a new criterion, referred as stability robustness, is introduced to estimate the sensitivity of those behaviors to disturbances of different levels, allowing to finely and accurately estimate suitable behaviors for energy harvesting purposes in realistic conditions (behaviors easy to reach and maintain in time). Experimental results conducted with a buckled beams based electromagnetic generator confirm the pertinence of this criterion showing good agreement with the analytical predictions. Subharmonic behaviors finally appears, both theoretically and experimentally, to be of significant interest, as exploiting them leads to a $180 \%$ increase of the global operating frequency range of the considered bistable energy harvester, for which more than $100 \mu \mathrm{W}$ are generated on a $70 \mathrm{~Hz}$ bandwidth at $0.5 \mathrm{~g}$.
\end{abstract}

\section{Introduction}

The race for energy harvesting in recent years is undoubtedly linked to the multiplication of stand-alone, left behind, wireless devices asking for more energetic autonomy that cannot be brought by primary batteries. More particularly, vibration have been highlighted as an interesting indoor/confined environment energy through the introduction of a wide variety of inertial harvesters composed of a spring-mass system coupled with electromagnetic or piezoelectric transducers [1]. Easy to implement on the vibration source, those harvesters can improve the wireless device compactness (no need to store and embed their entire lifetime energy) and reduce the maintenance costs (no need to replace empty batteries). The frequency bandwidth however remains one of the main limiting factors of inertial energy harvesters and their adaptability to non-constant or random vibrations is still a challenging issue.

Linear harvesters are indeed used to amplify ambient vibrations matching their natural frequency as studied by Williams et al. [2] but their performance dramatically drops in the close neighborhood of this particular frequency. Erturk et al. [3] first reported that nonlinear bistable harvesters show promising results with enhanced frequency bandwidth compared to linear harvesters [4-8]. Moreover, it is interesting to note that the classical method to determine the behaviors of bistable harvesters as a function of the ambient frequency does not reveal the entire richness of their spectra. Classical frequency sweep characterization may indeed miss behaviors as it only consists in smoothly and slowly increasing or decreasing the excitation frequency and recording the associated behaviors. As the nonlinear aspect of bistable harvesters allows different behaviors to coexist at the same frequency, those frequency-sweep behaviors may not be the only existing ones on the full frequency range. Hidden behaviors may then be used to magnify even more the already extended frequency bandwidth of bistable harvesters.

Among them, subharmonic behaviors have already been noticed in mechanical studies [9-12], but without any true exploitation. Arietta et al. [13] and Syta et al. [14] investigated the energy harvested on some subharmonic behaviors but did not evaluate their influence on the global frequency bandwidth of the harvester. However, the authors have already shown, in a purely experimental study, that the use of these behaviors could triple the useful frequency range of the bistable energy harvesters [15]. 
To confirm the observations made in the previous experimental study, this article first focuses on the analytical investigation of these original subharmonic behaviors, by analytically evaluating their contribution to the bandwidth enhancement of bistable harvesters. To deepen the analytical analysis, a novel stability criterion referred as stability robustness is introduced to estimate the sensitivity of those behaviors to disturbances of different levels. For high stability robustness, the behaviors will be considered suitable for energy harvesting purposes because they are easy to reach and maintain over time thanks to their low sensitivity to disturbances.

Some considerations on the robustness of behaviors of bistable oscillators have been already introduced by Lansbury et al. [16] who analyzed numerically the size of the basin of attraction of these behaviors (i.e., they analyzed the size of the area linked to the behavior under consideration on the numerical diagram showing the steady-state behavior reached depending on the initial conditions of the mass). The bigger the basin of attraction of the behavior, the easier it is to reach, and the more robust it is. Later, Harne et al. [17] also developed a robustness criterion for the most common behavior (first harmonic behavior) of bistable harvesters by simulating the effect of adding white noise on a sinusoidal excitation and checking if the behavior under consideration was maintained. In this paper, the approach proposed is still different: the stability robustness criterion is defined by the minimum amount of kinetic energy needed to destabilize the behavior. This minimum amount of kinetic energy is determined analytically following a mathematical method developed in the framework of differential equations known as bootstrap method [18, 19]. Moreover, the novel stability robustness criterion proposed here is calculated analytically contrary to criteria proposed by Lansbury et al. or Harne et al. and therefore requires a lower computing time, while, thanks to its semi-analytical formulation, would permit some optimization considerations.

The stability robustness is calculated for both first harmonic and subharmonic behaviors. While many works assessed the stability robustness by underestimating the mechanical quality factor, experimental results confirm that taking into account this stability robustness criteria is mandatory to predict the harmonic and subharmonic behaviors of a realistic bistable harvester.

\section{Problem statement}

\section{II.1. Mathematical model}

Figure 1 presents a common bistable oscillator configuration (other configurations can be found in the literature). The frame is submitted to the ambient vibration and the mass position is defined with respect to this frame.
The vibration energy harvester considered in this study is a bistable harvester composed of a generic bistable oscillator coupled with an electromagnetic transducer whose coil is linked to a load resistance for energy harvesting purposes. The electromagnetic transducer converts the kinetic energy of the mass into electrical energy which then dissipates in the load resistance by Joule's effect. The study focuses on this dissipated energy which represents the total energy converted by the bistable harvester. The mathematical model used to describe the bistable harvester is a Duffing-type mechanical equation coupled with the electromagnetic equation:

$$
\left\{\begin{array}{l}
\ddot{x}+\frac{\omega_{0}^{2}}{2}\left(\frac{x^{2}}{x_{0}^{2}}-1\right) x+\frac{\omega_{0}}{Q} \dot{x}+\frac{\beta}{M} I=-A \cos (\omega t) \\
R I=\beta \dot{x}-r_{L} I-L_{0} \dot{I}
\end{array}\right.
$$

With $M$ the mobile mass, $x$ its relative position with respect to the frame and $\pm x_{0}$ its relative stable position. $\omega_{0}$ is the natural angular frequency and $Q$ the mechanical quality factor of the equivalent linear oscillator obtained when the mass oscillates near one of the stable positions ( $x= \pm x_{0}+\Delta x$ with $\left.\Delta x<<\left|x_{0}\right|\right)$ as defined by Liu et al. [20]. $\omega$ and $A$ are angular frequency and the ambient acceleration. $\beta$ is the electromagnetic transducer equivalent force factor as defined by Arroyo et al. [21] and $r_{L}$ and $L_{0}$ are the coil internal resistance and inductance, respectively. $R$ is the load resistance connected to the coil and $I$ the current circulating through it. The values of the parameters used in this paper for simulation and analytic resolution correspond to the prototype presented in the experimental part and are listed in Table 1.

The coil impedance is $\sqrt{r_{L}^{2}+\left(\omega L_{0}\right)^{2}}$. For the frequency band under consideration $(20-200 \mathrm{~Hz})$, the term $\left(\omega L_{0}\right)^{2}$ is much lower compared to the term $r_{L}^{2}$ (respectively $39 \Omega^{2}$ and $324 \Omega^{2}$ at $200 \mathrm{~Hz}$ ). The coil impedance

\section{Buckled beam}
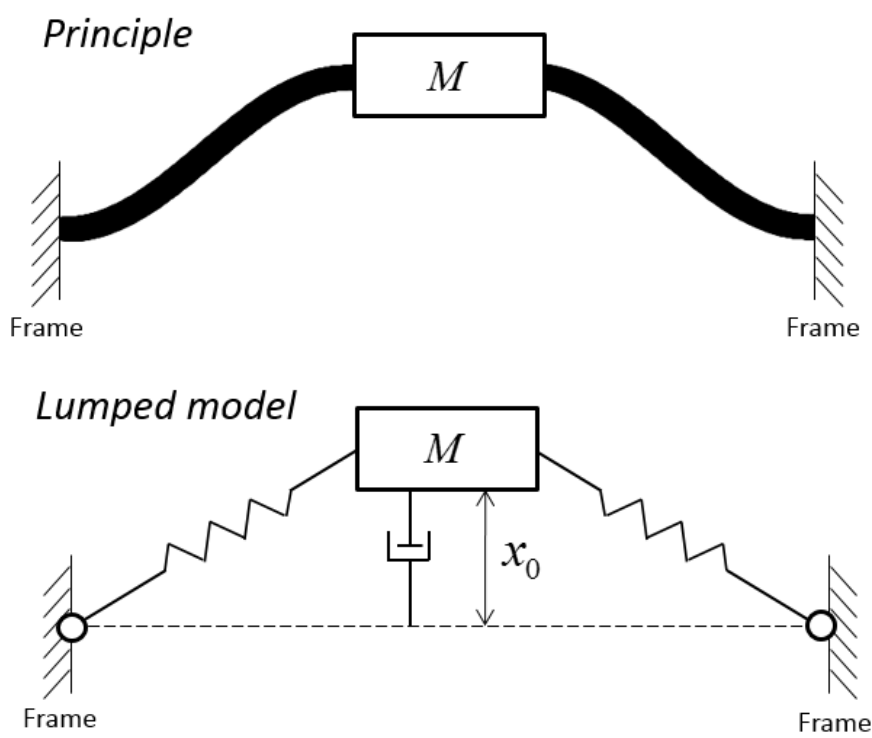

Figure 1: Principle and lumped model of a common bistable oscillator. 
Table 1: Parameter values of the bistable harvester prototype corresponding to the experimental prototype and used for numerical and analytical analysis.

\begin{tabular}{lccc}
\hline \hline Parameter & Symbol & Value & Unit \\
\hline Stable positions & $\pm x_{0}$ & \pm 0.29 & $\mathrm{~mm}$ \\
Inertial mass & $\mathrm{m}$ & 30 & $\mathrm{~g}$ \\
Natural angular frequency & $\omega_{0}$ & 229 & $\mathrm{rad}^{-1}$ \\
Mechanical quality factor & $Q$ & 113 & - \\
Excitation magnitude & $A$ & 5 & $\mathrm{~m}^{-2} \mathrm{~s}^{-2}$ \\
Excitation frequency & $\omega$ & $20-200$ & $\mathrm{~Hz}$ \\
Electromagnetic force factor & $\beta$ & 0.5 & $\mathrm{~N} . \mathrm{A}^{-1}$ \\
Coils internal resistance & $r_{L}$ & 18 & $\Omega$ \\
Coils inductance & $L_{0}$ & 5 & $\mathrm{mH}$ \\
Load resistance & $R$ & 18 & $\Omega$ \\
& & & \\
\hline \hline
\end{tabular}

is therefore considered as purely resistive and the inductance term in Equation (1) is neglected. The bistable harvester is then governed by the following single equation:

$$
\begin{aligned}
& -A \cos (\omega t)= \\
& \quad \ddot{x}+\frac{\omega_{0}^{2}}{2}\left(\frac{x^{2}}{x_{0}^{2}}-1\right) x+\left(\frac{\omega_{0}}{Q}+\frac{\beta^{2}}{M\left(R+r_{L}\right)}\right) \dot{x}
\end{aligned}
$$

The effect of the electromagnetic transducer is then equivalent to an additional damping as already suggested in previous studies [22]. The energy provided by the electromagnetic transducer is equivalent to the energy dissipated through this additional damping.

\section{II.2. Numerical examples of subharmonic behaviors}

Bistable harvesters, contrary to linear ones, can reach different steady-state behaviors (or orbits) depending on their initial conditions (mass position and mass velocity) for the same ambient vibration. Numerical resolutions of Equation (2) with different mass initial conditions yield the examples of the different orbits shown in Figure 2. The phase portrait is introduced for each orbit with its respective Poincaré map (black dots) and its time signal.

The mass oscillating around one stable position $\left(x_{0}\right.$ or $\left.-x_{0}\right)$, as shown in Figure 2(a), corresponds to low orbits (intra-well behavior). The mass oscillating from a stable position to the other (i.e. regularly crossing the $x=0$ position), as shown in Figure 2(b), corresponds to high orbits (inter-well behavior). More particularly, the harmonic 1 orbit is reached when the mass oscillates at the same frequency as the excitation as detailed in Figure 2(a) (harmonic 1 low orbit) and Figure 2(b) (harmonic 1 high orbit). These harmonic 1 orbits are well known as they naturally appear using frequency sweep characterization. Indeed, those orbits have the particularity to be the only possible behaviors of the bistable generator on certain frequency ranges and thus, are automatically reached during a frequency sweep. On the opposite, the subharmonic orbits shown in Figure 2(c) to (f) do not naturally appear during a frequency sweep and need a jump phenomenon to be reached. This can be done, for instance, through a change in the mass initial conditions. Subharmonic $n$ orbit is reached when the mass moves $n$ times slower than the excitation. In Figure 2, only high orbits are presented for subharmonic behaviors despite the existence of low orbits, as low orbits are not of interest for energy harvesting due to their low amplitudes and associated energies.
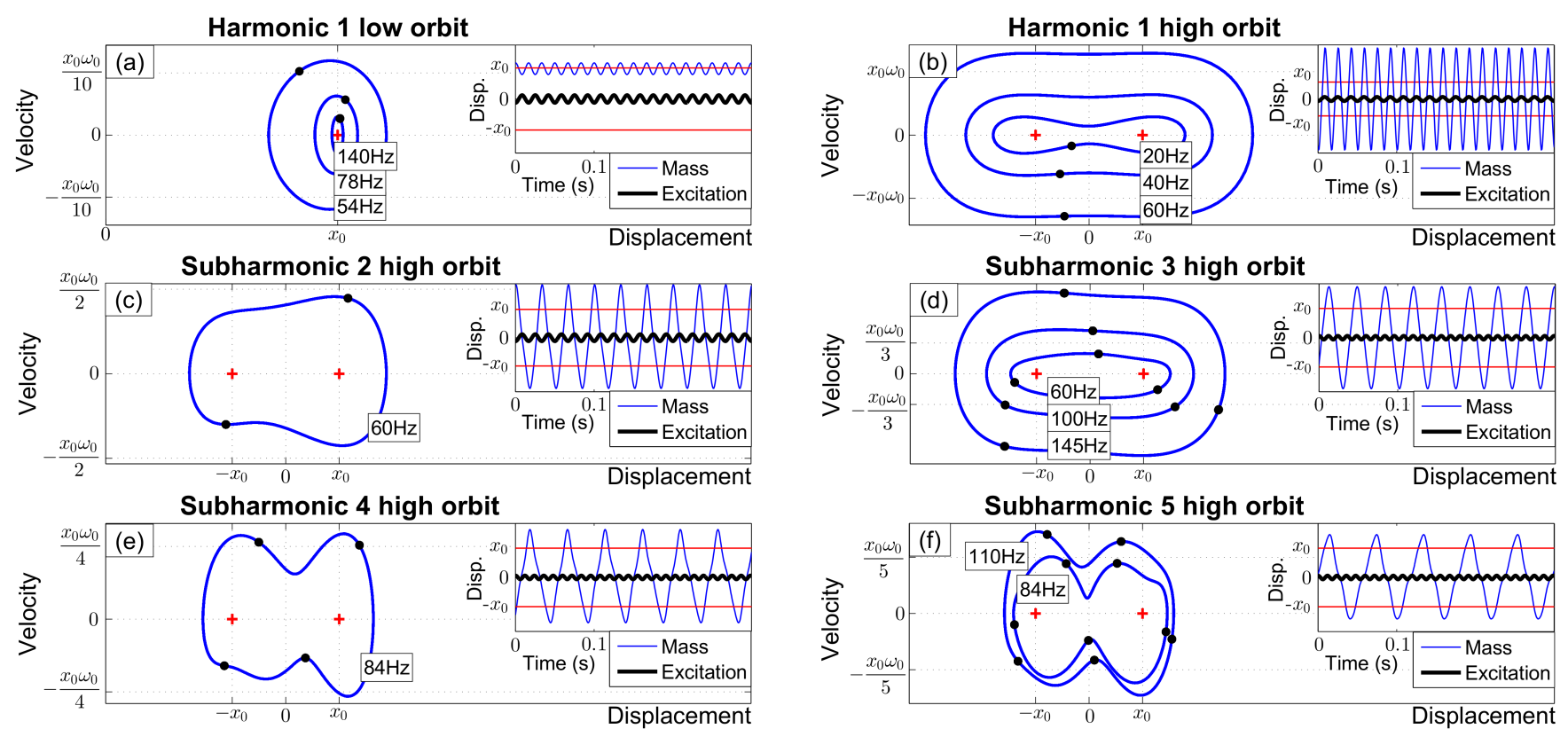

Figure 2: Numerical examples of different behaviors (or orbits) of bistable harvesters including subharmonic orbits for which the displacement frequency is lower than the excitation frequency ( $n$ times lower for subharmonic $n$ ). For each orbit, a phase portrait with its Poincare map (black dots) as well as a time signal is presented. 


\section{Analytical analysis}

Subharmonic high orbits, appearing on certain frequency ranges, could be therefore exploited to widen the frequency bandwidth of bistable harvesters and thus magnify the principal asset of nonlinear vibrational energy harvesters. This section presents the analytical investigation of the different steady-state behaviors of bistable harvesters governed by Equation (2) to evaluate the frequency bandwidth of bistable harvesters including subharmonic orbits. This analytical investigation leads to the bistable generator spectrum containing all its possible steady-state behaviors as a function of the ambient vibration frequency. For the sake of easier readability, vectors are underlined once and matrices underlined twice.

\section{III.1. Steady-state behaviors}

The followings present the global methodology to analytically solve Equation (2) with the Harmonic Balance method for the purpose of describing the harmonic and subharmonic behaviors. First, several assumptions have to be made for obtaining Equation (2) solutions: (1) the bistable harvester is in steady-state motion; (2) the response is approached by a truncated Fourier series of order $N$; (3) the behavior under consideration is the subharmonic $n$ orbit. With $\omega$ the excitation pulsation, the response is hence composed by a constant term and a preponderant pulsation $\omega / n$ followed by its harmonics $k \omega / n$ with $k$ varying from 2 to $N$. The general response of Equation (2) is then assumed as:

$$
x(t)=a_{0}+\sum_{k=1}^{N}\left\{a_{k} \cos \left(k \frac{\omega}{n} t\right)+b_{k} \sin \left(k \frac{\omega}{n} t\right)\right\}
$$

The constant term $a_{0}$ corresponds to the mean displacement. If $a_{0}$ is close to zero, the solution is a high orbit. If $a_{0}$ is close to $x_{0}$ or $-x_{0}$ (stable positions), the solution is a low orbit. The system is in a steady-state motion, hence, the $2 N+1$ unknowns $\left(a_{0}, a_{k}, b_{k}\right), k \in \llbracket 1, N \rrbracket$ are constant. Substituting Equation (3) into Equation (2) leads to Equation (4) composed by a sum of sinuses and cosines of different frequencies.

In Equation (4), $b_{0}=0$ is added to simplify the global expression. The terms composing Equation (4) correspond to the different forces acting at different frequencies on the mass and the equation describes how they equilibrate. In the truncated response form described in Equation (3), the terms with a pulsation higher than $N \omega / n$ are considered as negligible. Similarly, in Equation (4), the force terms with a pulsation higher than $N \omega / n$ are also considered as negligible leaving $2 N+1$ terms: a constant term, $N$ sinus terms and $N$ cosine terms. The Harmonic Balance method is then applied to these $2 \mathrm{~N}+1$ force terms so that they are all equated to zero one by one leading to a solvable nonlinear system made of $2 N+1$ equations and $2 N+1$

$$
\begin{aligned}
& \sum_{k \in \llbracket 0, N \rrbracket}\{ \\
& {\left[\left(k \frac{\omega}{n}\right)^{2} a_{k}+\frac{\omega_{0}^{2}}{2} a_{k}-\left(\frac{\omega_{0}}{Q}+\frac{\beta^{2}}{M\left(R+r_{L}\right)}\right) k \frac{\omega}{n} b_{k}\right] \cos \left(k \frac{\omega}{n} t\right) } \\
+ & {\left.\left[\left(k \frac{\omega}{n}\right)^{2} b_{k}+\frac{\omega_{0}^{2}}{2} b_{k}+\left(\frac{\omega_{0}}{Q}+\frac{\beta^{2}}{M\left(R+r_{L}\right)}\right) k \frac{\omega}{n} a_{k}\right] \sin \left(k \frac{\omega}{n} t\right)\right\} } \\
- & \sum_{(i, k, s) \in \llbracket 0, N \rrbracket} \frac{1}{8} \frac{\omega_{0}^{2}}{x_{0}^{2}}\{ \\
& \left(a_{i} a_{k} a_{s}-a_{i} b_{k} b_{s}-b_{i} a_{k} b_{s}-b_{i} b_{k} a_{s}\right) \cos \left((i+k+s) \frac{\omega}{n} t\right) \\
+ & \left(a_{i} a_{k} a_{s}+a_{i} b_{k} b_{s}+b_{i} a_{k} b_{s}-b_{i} b_{k} a_{s}\right) \cos \left((i+k-s) \frac{\omega}{n} t\right) \\
+ & \left(a_{i} a_{k} a_{s}+a_{i} b_{k} b_{s}-b_{i} a_{k} b_{s}+b_{i} b_{k} a_{s}\right) \cos \left((i-k+s) \frac{\omega}{n} t\right) \\
+ & \left(a_{i} a_{k} a_{s}-a_{i} b_{k} b_{s}+b_{i} a_{k} b_{s}+b_{i} b_{k} a_{s}\right) \cos \left((i-k-s) \frac{\omega}{n} t\right) \\
+ & \left(b_{i} a_{k} a_{s}+a_{i} a_{k} b_{s}+a_{i} b_{k} a_{s}-b_{i} b_{k} b_{s}\right) \sin \left((i+k+s) \frac{\omega}{n} t\right) \\
+ & \left(b_{i} a_{k} a_{s}-a_{i} a_{k} b_{s}+a_{i} b_{k} a_{s}+b_{i} b_{k} b_{s}\right) \sin \left((i+k-s) \frac{\omega}{n} t\right) \\
+ & \left(b_{i} a_{k} a_{s}+a_{i} a_{k} b_{s}-a_{i} b_{k} a_{s}+b_{i} b_{k} b_{s}\right) \sin \left((i-k+s) \frac{\omega}{n} t\right) \\
+ & \left.\left(b_{i} a_{k} a_{s}-a_{i} a_{k} b_{s}-a_{i} b_{k} a_{s}-b_{i} b_{k} b_{s}\right) \sin \left((i-k-s) \frac{\omega}{n} t\right)\right\} \\
- & A \cos (\omega t)=0
\end{aligned}
$$

unknowns. For a given set of bistable harvester's parameters $\left(M, x_{0}, \omega_{0}, Q, \beta, r_{L}, R\right)$ and for a given ambient vibration $A \cos (\omega t)$, this system may present more than one solution due to its nonlinearity. Each solution represents a possible steady-state behavior (or orbit) for the bistable harvester. The $m^{\text {th }}$ solution (or the $m^{\text {th }}$ behavior or the $m^{\text {th }}$ orbit) can then be represented by a $2 N+1$ cells array noted as:

$$
\underline{S_{m}}=\left(a_{m 0}, a_{m k}, b_{m k}\right)_{k \in \llbracket 1, N \rrbracket}
$$

with $x_{m}(t)=a_{m 0}+\sum_{k=1}^{N}\left\{a_{m k} \cos \left(k \frac{\omega}{n} t\right)+b_{m k} \sin \left(k \frac{\omega}{n} t\right)\right\}$

$x_{m}$ is the temporal signal of the $m^{\text {th }}$ steady-state behavior of the bistable harvester for the ambient vibration $A \cos (\omega t)$. This procedure, performed for different ambient vibration frequencies $\omega$, leads to the bistable harvester spectrum containing all the different steady-state behaviors $x_{m}$ as a function of the ambient vibration frequency.

\section{III.2. Disturbances stability analysis}

Subsection 1 established the possible analytical solutions for the steady-state behaviors of the bistable harvester. However, the proposed method does not permit evaluating their stability. Hence, a stability analysis with a small perturbation method is performed on all the possible 
steady-state behaviors $x_{m}$. For this purpose, the response is considered to be composed of 2 different terms: the steady-state behavior under study $x_{m}$ and a transient term $z$ reflecting the disturbance, considered small in magnitude compared to $x_{m}$.

$$
\left\{\begin{array}{l}
x(t)=x_{m}(t)+z(t) \\
\|z(t)\|<<\left\|x_{m}(t)\right\|
\end{array}\right.
$$

The steady-state behaviors $x_{m}$ is stable if $z$ tends toward zero with time. Equation (6) introduced in Equation (2) and linearized for small $z$ leads to the disturbance statespace equations of motion:

$$
\left(\begin{array}{c}
\dot{z}(t) \\
\ddot{z}(t)
\end{array}\right)=\underline{\underline{M}}(t)\left(\begin{array}{l}
z(t) \\
\dot{z}(t)
\end{array}\right)
$$

with $\underline{\underline{M}}(t)=\left[\begin{array}{cc}0 & 1 \\ \frac{\omega_{0}^{2}}{2}-\frac{3 \omega_{0}^{2}}{2 x_{0}^{2}} x_{m}(t)^{2} & -\frac{\omega_{0}}{Q}-\frac{\beta^{2}}{M\left(R+r_{L}\right)}\end{array}\right]$

Where $\underline{M}(t) \in M_{2,2}(\mathbb{R})$ is $T$-periodic with $T=n / \omega$. The resolvent matrix $\underline{\underline{R}}\left(t, t_{0}\right) \in M_{2,2}(\mathbb{R})$ of Equation (7) is introduced. It gives the relationship between the disturbance at $t_{0}$ (initial conditions) and the disturbance at $t$ following:

$$
\begin{aligned}
\left(\begin{array}{l}
z(t) \\
\dot{z}(t)
\end{array}\right)=\underline{\underline{R}}\left(t, t_{0}\right) & \left(\begin{array}{l}
z\left(t_{0}\right) \\
\dot{z}\left(t_{0}\right)
\end{array}\right) \\
\text { with } & \left\{\begin{array}{l}
\frac{\partial \underline{\underline{R}}}{\partial t}\left(t, t_{0}\right)=\underline{\underline{M}}(t) \underline{\underline{R}}\left(t, t_{0}\right) \\
\underline{\underline{R}}\left(t+T, t_{0}+T\right)=\underline{\underline{R}}\left(t, t_{0}\right)
\end{array}\right.
\end{aligned}
$$

With $\underline{\underline{R}}\left(t, t_{0}\right) \in M_{2,2}(\mathbb{R})$. This resolvent matrix can be numerically calculated for all $t$. Indeed, a numerical integration of Equation (7) from $t_{0}$ to $t$ for 2 initial conditions corresponding to the 2 vectors of the standard basis of $\mathbb{R}^{2}$ leads to 2 vectors which completely define the resolvent matrix:

$$
\begin{aligned}
& \left(\begin{array}{l}
z_{1}\left(t_{0}\right) \\
z_{1}\left(t_{0}\right)
\end{array}\right)=\left(\begin{array}{l}
1 \\
0
\end{array}\right) \underset{\text { from } t_{0} \text { to } t \text { of Equation }(7)}{\stackrel{\text { numerical integration }}{\longrightarrow}}\left(\begin{array}{l}
z_{1}(t) \\
z_{1}(t)
\end{array}\right) \\
& \left.\left(\begin{array}{l}
z_{2}\left(t_{0}\right) \\
\dot{z_{2}}\left(t_{0}\right)
\end{array}\right)=\left(\begin{array}{l}
0 \\
1
\end{array}\right) \underset{\text { from } t_{0} \text { to } t \text { of Equation }(7)}{\stackrel{\text { numerical integration }}{\longrightarrow}}\left(\begin{array}{l}
z_{2}(t) \\
\dot{z_{2}}(t)
\end{array}\right)\right\} \\
& \Rightarrow \underline{\underline{R}}\left(t, t_{0}\right)=\left[\left(\begin{array}{l}
z_{1}(t) \\
\dot{z}_{1}(t)
\end{array}\right)\left(\begin{array}{l}
z_{2}(t) \\
\dot{z_{2}}(t)
\end{array}\right)\right]
\end{aligned}
$$

$\underline{M}(t) \in M_{2,2}(\mathbb{R})$ is $T$-periodic so the Floquet Theory [23] can be applied. Hence, it is possible to define a constant matrix $\underline{B} \in M_{2,2}(\mathbb{R})$ and a $2 T$-periodic matrix $\underline{\underline{Q}}\left(t, t_{0}\right) \in M_{2,2}(\mathbb{R})$ such that:

$$
\underline{\underline{R}}\left(t, t_{0}\right)=\underline{\underline{Q}}\left(t, t_{0}\right) e^{-\left(t-t_{0}\right) \underline{\underline{B}}}
$$

The 2 complex eigenvalues of $\underline{\underline{B}}$ are noted $\left(\lambda_{1}, \lambda_{2}\right)$. Assuming $\underline{B}$ is invertible, a constant and invertible matrix $\underline{\underline{P}} \in M_{2,2}(\mathbb{C})$ can be defined such that:

$$
\underline{\underline{R}}\left(t, t_{0}\right)=\underline{\underline{Q}}\left(t, t_{0}\right) \underline{\underline{P}}^{-1}\left(\begin{array}{cc}
e^{-\lambda_{1}\left(t-t_{0}\right)} & 0 \\
0 & e^{-\lambda_{2}\left(t-t_{0}\right)}
\end{array}\right) \underline{\underline{P}}
$$

As $\underline{Q}\left(t, t_{0}\right)$ is $2 T$-periodic and $\underline{\underline{P}}$ is constant, then the stability condition of the steady-state behavior $x_{m}$ is directly linked to the eigenvalues of $\underline{\underline{B}}$ namely $\left(\lambda_{1}, \lambda_{2}\right)$ :

$$
x_{m} \text { stable } \Leftrightarrow\left\{\begin{array}{l}
\operatorname{Re}\left(\lambda_{1}\right)>0 \\
\operatorname{Re}\left(\lambda_{2}\right)>0
\end{array}\right.
$$

Moreover, $\underline{\underline{B}}$ is directly linked to the resolvent matrix after a $2 T$ integration:

$$
\begin{aligned}
\underline{\underline{R}}(2 T, 0) & =\underline{\underline{Q}}(2 T, 0) e^{-2 T \underline{\underline{B}}} \\
& =\underline{\underline{Q}}(0,0) e^{-2 T \underline{\underline{B}}} \\
& =e^{-2 T \underline{\underline{B}}}
\end{aligned}
$$

With $\underline{R}(2 T, 0)$ calculable following Equation (9). The 2 complex eigenvalues of $\underline{\underline{R}}(2 T, 0)$ are noted $\left(\beta_{1}, \beta_{2}\right)$ and verify:

$$
\left\{\begin{array}{l}
\beta_{1}=e^{-2 \lambda_{1}} \\
\beta_{2}=e^{-2 \lambda_{2}}
\end{array}\right.
$$

The condition of stability of $x_{m}$ previously related to $\left(\lambda_{1}, \lambda_{2}\right)$ as stated in Equation (12) can hence be defined with the eigenvalues of $\underline{\underline{R}}(2 T, 0)$ namely $\left(\beta_{1}, \beta_{2}\right)$ :

$$
x_{m} \text { stable } \Leftrightarrow\left\{\begin{array}{l}
\left|\beta_{1}\right|<1 \\
\left|\beta_{2}\right|<1
\end{array}\right.
$$

In conclusion, the stability of a steady-state behavior $x_{m}$ is determined by the eigenvalues of the resolvent matrix evaluated at $\left(t=2 T, t_{0}=0\right)$ thanks to a numerical integration of Equation (7) from 0 to $2 T$ for 2 initial conditions corresponding to the 2 vectors of the standard basis of $\mathbb{R}^{2}$.

\section{III.3. Stability: analytical results}

Figure 3 introduces the bistable harvester spectra obtained with the Harmonic Balance method and the stability analysis described previously for the parameter values listed in Table 1. The Fourier series responses are truncated to $N=6$ (the energy associated to the higher harmonics being negligible compared to the lower ones). Figure 3(a) details the mass displacement amplitude with respect to the support for the different steady-state orbits as a function of the ambient vibration frequency. Figure $3(\mathrm{~b})$ details the phase of the excitation displacement when the position of the mass reaches a maximum. Figure 3(c) shows the average harvested power only on the stable orbits through the resistance $R$; the latter being chosen to match the transducer internal resistance to maximized energy transfer. The expressions used to calculate the data in those figures are detailed in Appendix A.

Six different behaviors are visible in Figure 3(a): one low orbit (motion amplitude inferior to $x_{0}$ ) and five high orbits (motion amplitudes superior to $x_{0}$ ). As low orbits are not interesting for vibration energy harvesting 

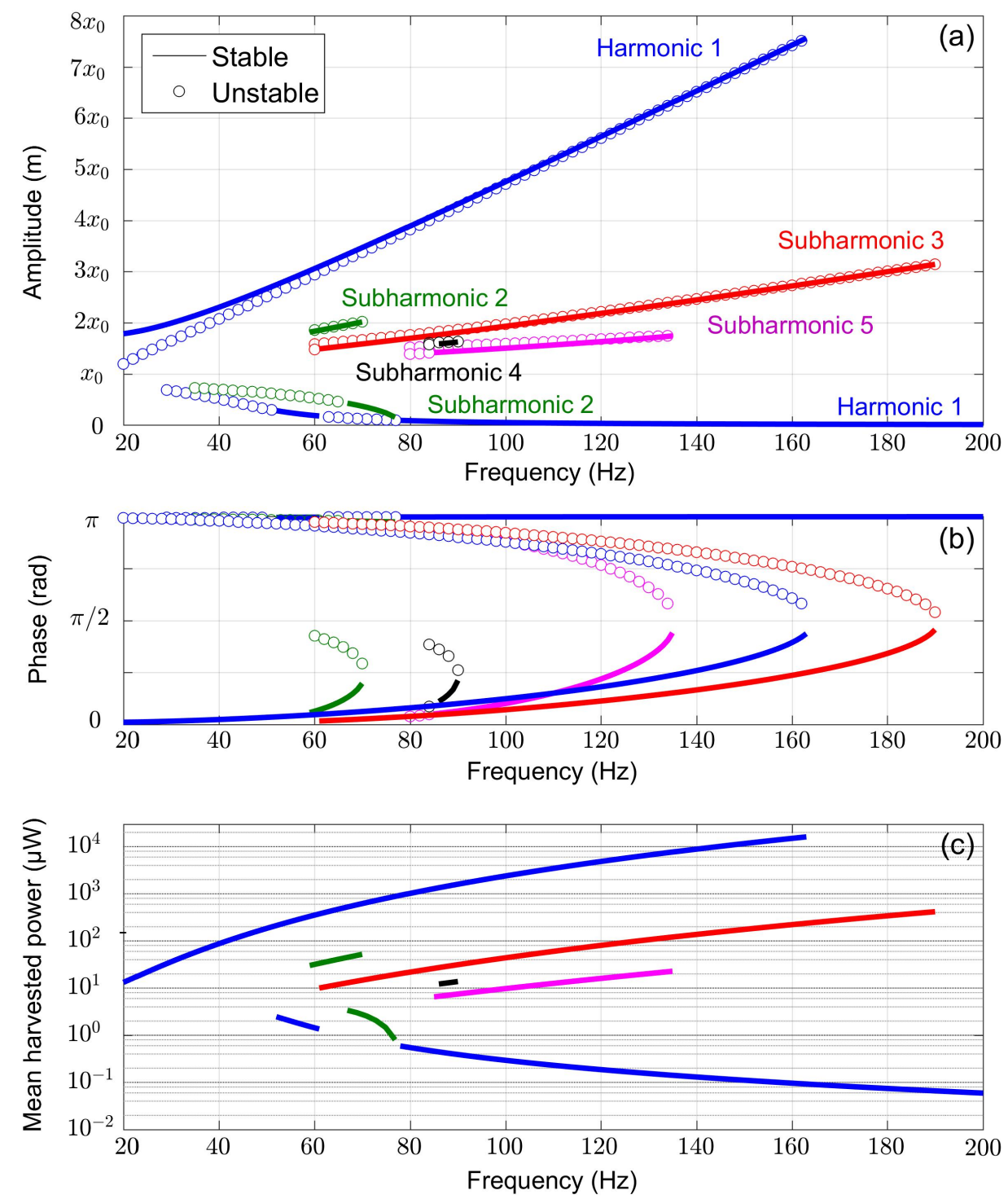

Figure 3: Bistable harvester analytical spectra for an excitation amplitude of $5 \mathrm{~m} \cdot \mathrm{s}^{-2}$ : (a) orbits' amplitude, (b) phase of the excitation displacement when the position of the mass reaches a maximum and (c) average power harvested for stable orbits.

compared to high orbits, only the harmonic 1 low orbit is depicted as reference. The high orbit list is also non-exhaustive. Considering that subharmonic high orbit amplitude globally decreases with its order (subharmonic $n+1$ amplitude is smaller than subharmonic $n$ amplitude), the subharmonic high orbits with low orders are more interesting for energy harvesting. For this reason, Figure 3 does not present subharmonic orbits higher than order 5 .

From these analytical results, it is interesting to note that the spectrum of the bistable harvester is much richer compared to the spectrum obtained with a frequency sweep characterization. Indeed, the frequency sweep method, too smooth to allow orbit changes, only highlights the behaviors existing alone on certain frequency ranges. Figure 3(a) confirms that the orbits presenting this particularity are the most studied orbits, i.e., harmonic 1 high orbit and harmonic 1 low orbit. All of the subharmonics orbits are always coexisting with one of these common orbits on their whole frequency range and thus are not caught with a frequency sweep characterization.

The phase of the excitation displacement when the position of the mass reaches a maximum presented in Figure 3(b) also suggests an interesting characteristic of bistable oscillators. Indeed, for odd high orbits, it increases monotonously with ambient vibration frequency until $\pi / 2$ which corresponds to the orbit cutting frequency. For even high orbits, it also increases monotonously with ambient vibration frequency until $\pi / 4$ which corresponds to the orbit cutting frequency. This particularity has been first reported for the harmonic 1 orbit by Harne et al. [24] and is here extended to subharmonic behaviors. An analogy can be made with linear oscillators which also present an increasing phase until $\pi / 2$, which corresponds to the resonance peak. However, in the case of linear oscillators, the phase then keeps on increasing and tends toward $\pi$ without 
any discontinuity (i.e., without cutting frequency). This aspect of bistable oscillators is helpful for experimental characterization as it may confirm or not if the theoretical high orbits cutting frequency has been effectively reached during the experiments.

Finally, it can be seen in Figure 3(a) that subharmonic 3 frequency range is not entirely included in harmonic 1 high orbit's one. Thus, this behavior is a good candidate to enhance the global frequency bandwidth of the bistable harvester. However, when considering orbit stability only, the average harvested power on subharmonic 3 behavior (with a maximum of $416 \mu \mathrm{W}$ ) is relatively small compared to harmonic 1 behavior (with a maximum of $1.61 \mathrm{~mW}$ ).

\section{A novel criterion: the stability robustness}

Previous results indicates that exploiting subharmonic behaviors may not be so interesting since the harvestable power on harmonic 1 orbit is theoretically much larger than subharmonics one, and the bandwidth enhancement offered by subharmonic 3 is rather limited $(15 \mathrm{~Hz}$ extension). However, experiments show that the stable high orbits are not all equivalent, being more or less easy to reach and maintain. The orbit stability criteria defined in the previous section is calculated for a small disturbance inducing infinitesimal variation around the steady-state response. But, among stable high orbits, when the disturbance is increased, some remain stable and others become unstable, which actually induces a drop to harmonic 1 low orbit. In particular, the maximal power point of the harmonic 1 response cannot be practically reached. In order to take into account this phenomenon, a new criterion is analytically introduced and presented, namely the stability robustness. It indicates the sensitivity of stable high orbits to disturbances of different levels. Hence, for low stability robustness, the high orbit will no longer be considered as suitable for energy harvesting because of the difficulty to reach and maintain over time. In this section, the stability robustness is calculated for all the steady-state high orbits $x_{m}$ defined as stable with the small disturbances criterion defined in the previous section.

The idea of the stability robustness analysis is to disturb the stable high orbit with a certain amount of energy (added or removed to the system). Then, the minimum of this energy needed to destabilize the high orbit determine its robustness. The greater this energy, the more robust the orbit. The energy used to disturb the steadystate high orbit is a kinetic energy defined by a speed $\Lambda$ added to the speed of the mass at $t=t_{0}$ ( $\Lambda$ can be positive or negative). Similarly to the previous section, the response is decomposed into 2 signals: the steady-state high orbits $x_{m}$ and a transient term $z$ reflecting the disturbance. The speed added to the speed of the mass is then represented by the initial conditions of the disturbance signal z:

$$
\left(\begin{array}{l}
z\left(t_{0}\right) \\
\dot{z}\left(t_{0}\right)
\end{array}\right)=\left(\begin{array}{c}
0 \\
\Lambda
\end{array}\right)
$$

If $\left|\Lambda_{\min }\right|$ is the minimum speed leading to instability, the stability robustness is defined as the corresponding kinetic energy normalized by the mean energy brought by the excitation to the mass per period of excitation:

$$
\begin{aligned}
\text { Robustness } & =\frac{\frac{1}{2} M \Lambda_{\min }^{2}}{E_{\text {excitation }}} \\
\text { with } & E_{\text {excitation }}=-\frac{1}{n} \int_{0}^{T} M A \cos (\omega t) \dot{x}_{m} d t
\end{aligned}
$$

With $T$ the period of the subharmonic $n$ behavior $x_{m}$ under study. The followings present the method leading to the determination of $\left|\Lambda_{\text {min }}\right|$ which is based on a mathematical method known as bootstrap method [18, 19]. In the same way as in the previous section, Equation (6) is introduced in Equation (2) but $z$ is not anymore considered as small compared to $x_{m}$ so that linearization cannot be considered. Then, the disturbance state-space equation of motion becomes:

$$
\left(\begin{array}{l}
\dot{z}(t) \\
\ddot{z}(t)
\end{array}\right)=\underbrace{\underline{\underline{M}}(t)\left(\begin{array}{c}
z(t) \\
\dot{z}(t)
\end{array}\right)}_{\text {linear term }}+\underbrace{\left(\begin{array}{c}
0 \\
-\frac{3 \omega_{0}^{2}}{2 x_{0}^{2}} x_{m}(t) z(t)^{2}-\frac{\omega_{0}^{2}}{2 x_{0}^{2}} z(t)^{3}
\end{array}\right)}_{\text {non linear term }}
$$

Note that the evolution of the disturbance $z$ is linked to $x_{m}$ through the matrix $\underline{M}$. Thus, for all $t$, the disturbance state can be written as follow:

$$
\begin{aligned}
\left(\begin{array}{c}
z(t) \\
\dot{z}(t)
\end{array}\right)= & \underline{\underline{R}}\left(t, t_{0}\right)\left(\begin{array}{c}
0 \\
\Lambda
\end{array}\right) \\
& +\int_{t_{0}}^{t} \underline{\underline{R}}(t, s)\left(-\frac{3 \omega_{0}^{2}}{2 x_{0}^{2}} x_{m}(s) z(s)^{2}-\frac{\omega_{0}^{2}}{2 x_{0}^{2}} z(s)^{3}\right) d s
\end{aligned}
$$

With $\underline{\underline{R}}\left(t, t_{0}\right)$ defined by Equation (8) and Equation (10). As the steady-state high orbits $x_{m}$ studied here are stable with the small disturbances criterion, hence the two complex eigenvalues $\left(\lambda_{1}, \lambda_{2}\right)$ of $\underline{B}$ have positive real parts as stated in Equation (12). The variable $\lambda$ is introduced as the minimum of $\left(\lambda_{1}, \lambda_{2}\right)$ real parts:

$$
\lambda=\min \left(\operatorname{Re}\left(\lambda_{1}\right), \operatorname{Re}\left(\lambda_{2}\right)\right)
$$

A reductio ad absurdum is conducted to determine $\Lambda_{\min }$ considering the following hypothesis $H$ :

$$
\begin{aligned}
& H=\forall \delta>0, \exists \tau \geqslant t_{0}: \underbrace{\left(\forall t \in\left[t_{0}, \tau\right],|z(t)| \leqslant \delta e^{-\lambda\left(t-t_{0}\right)}\right)}_{H 1} \\
& \cap \underbrace{(\forall t \in] \tau,+\infty\left[,|z(t)|>\delta e^{-\lambda\left(t-t_{0}\right)}\right)}_{H 2}
\end{aligned}
$$


$H$ true implies $H 2$ true which implies that the disturbance tends toward infinity with time meaning that the steady-state behavior is unstable for $\Lambda$. The negation of $H$ is:

$$
\begin{aligned}
& \bar{H}=\exists \delta>0: \forall \tau \geqslant t_{0} \underbrace{\left(\exists t \in\left[t_{0}, \tau\right]:|z(t)|>\delta e^{-\lambda\left(t-t_{0}\right)}\right)}_{H 3} \\
& \cup \underbrace{(\exists t \in] \tau,+\infty\left[:|z(t)| \leqslant \delta e^{-\lambda\left(t-t_{0}\right)}\right)}_{H 4}
\end{aligned}
$$

$H 3$ is always wrong for $\tau=t_{0}$. Hence $H$ wrong implies $\mathrm{H} 4$ true which implies in turn that the disturbance tends toward zero with time hence meaning that the steadystate behavior is stable for $\Lambda$. In the following, $H$ is assumed as true. From the disturbance continuity, it can be deducted that:

$$
|z(\tau)|=\delta e^{-\lambda\left(\tau-t_{0}\right)}
$$

On another hand, all the components of the resolvent matrix $e^{\lambda\left(t-t_{0}\right)} \underline{\underline{R}}\left(t, t_{0}\right)$ can be upper bounded independently of time:

$$
\underline{\underline{R}}\left(t, t_{0}\right)=\underline{\underline{Q}}\left(t, t_{0}\right) \underline{\underline{P}}^{-1}\left(\begin{array}{cc}
e^{-\lambda_{1}\left(t-t_{0}\right)} & 0 \\
0 & e^{-\lambda_{2}\left(t-t_{0}\right)}
\end{array}\right) \underline{\underline{P}}
$$

Thus,

$$
\begin{aligned}
& e^{\lambda\left(t-t_{0}\right)} \underline{\underline{R}}\left(t, t_{0}\right)= \\
& \underbrace{\underline{Q}\left(t, t_{0}\right)}_{2 T \text { periodic }} \underbrace{\underline{P}^{-1}\left(\begin{array}{cc}
e^{\left(\lambda-\lambda_{1}\right)\left(t-t_{0}\right)} & 0 \\
0 & e^{\left(\lambda-\lambda_{2}\right)\left(t-t_{0}\right)}
\end{array}\right) \underline{\underline{P}}}_{\text {Periodic or decreasing with time }}
\end{aligned}
$$

Hence, for a given $t_{0}$, each component of the matrix $e^{\lambda\left(t-t_{0}\right)} \underline{R}\left(t, t_{0}\right)$ admits a maximum which occurs for $t \in$ $\left[t_{0} ; t_{0}+2 T\right]$. Using the periodic propriety of the resolvent matrix $\underline{\underline{R}}\left(t+T, t_{0}+T\right)=\underline{\underline{R}}\left(t, t_{0}\right)$ seen in Equation (8) then each component of the matrix $e^{\lambda\left(t-t_{0}\right)} \underline{\underline{R}}\left(t, t_{0}\right)$ admits a maximum which occurs for $t \in\left[t_{0} ; t_{0}+\overline{2} T\right]$ with $t_{0} \in$ $[0 ; T]$. Such a consideration leads to:

$$
\begin{aligned}
& \forall\left(t, t_{0}\right), \forall(i, j) \in \llbracket 1 ; 2 \rrbracket^{2}, \\
& {\left[\underline{\underline{R}}\left(t, t_{0}\right)\right]_{(i, j)} \leqslant e^{-\lambda\left(t-t_{0}\right)}[\underline{\underline{C}}]_{(i, j)} }
\end{aligned}
$$

With

$$
[\underline{\underline{C}}]_{(i, j)}=\max _{\substack{t_{0} \in[0, T] \\ t \in\left[t_{0}, t_{0}+2 T\right]}}\left|e^{\lambda\left(t-t_{0}\right)}\left[\underline{\underline{R}}\left(t, t_{0}\right)\right]_{(i, j)}\right|
$$

$\underline{\underline{C}} \in M_{2,2}(\mathbb{C})$ is a constant matrix independent from $t$ and $\overline{\overline{t_{0}}}$ and can be calculated from Equation (9). Equation (19) evaluated at $\tau$ yields:

$$
\begin{aligned}
& z(\tau)=\Lambda\left[\underline{\underline{R}}\left(\tau, t_{0}\right)\right]_{(1,2)}+ \\
& \int_{t_{0}}^{\tau}[\underline{\underline{R}}(\tau, s)]_{(1,2)}\left(-\frac{3 \omega_{0}^{2}}{2 x_{0}^{2}} x_{m}(s) z(s)^{2}-\frac{\omega_{0}^{2}}{2 x_{0}^{2}} z(s)^{3}\right) d s
\end{aligned}
$$


disturbance $\left|\Lambda_{\min }\right|$ respecting $f\left(\delta_{0}, \Lambda_{\min }=0\right)$. Hence the stability robustness criterion is defined as:

$$
\text { Robustness }=\frac{\frac{1}{2} M \Lambda_{\min }^{2}}{E_{\text {excitation }}}
$$

With

$$
\left\{\begin{array}{l}
E_{\text {excitation }}=-\frac{1}{n} \int_{0}^{T} M A \cos (\omega t) \dot{x}_{m} d t \\
\Lambda_{\min }=\frac{\delta_{0}-B \delta_{0}^{2}-D \delta_{0}^{3}}{[\underline{\underline{C}}]_{(1,2)}}
\end{array}\right.
$$

$\Lambda_{\text {min }}$ is the minimum speed disturbance inducing instability of the stable high orbit under consideration, $E_{\text {excitation }}$ is the mean energy brought by the excitation to the mass per period of excitation and $T$ is the period of the subharmonic $n$ behavior $x_{m}$ under study. The stable high orbits with a low stability robustness become unstable and drop to harmonic 1 low orbit for low energetic disturbances and thus are harder to reach and maintain over time. This criterion, which quantify the robustness of the stability for bistable harvesters is therefore useful to evaluate if the high orbits defined as stable with the common small disturbances criterion are easy to reach and maintain leading to their energy harvesting suitability.

Figure 4(c) introduces the stability robustness calculated for the stable high orbits exposed in the previous section. Those orbits are recalled in Figure 4(a) with the amplitude spectrum and in Figure 4(b) with the phase of the excitation displacement when the position of the mass reaches a maximum.

The stability robustness presented confirms the experimental observations: the stable high orbits are not all equally sensitive to disturbances. It can be seen that even subharmonic behaviors are far less robust than odd
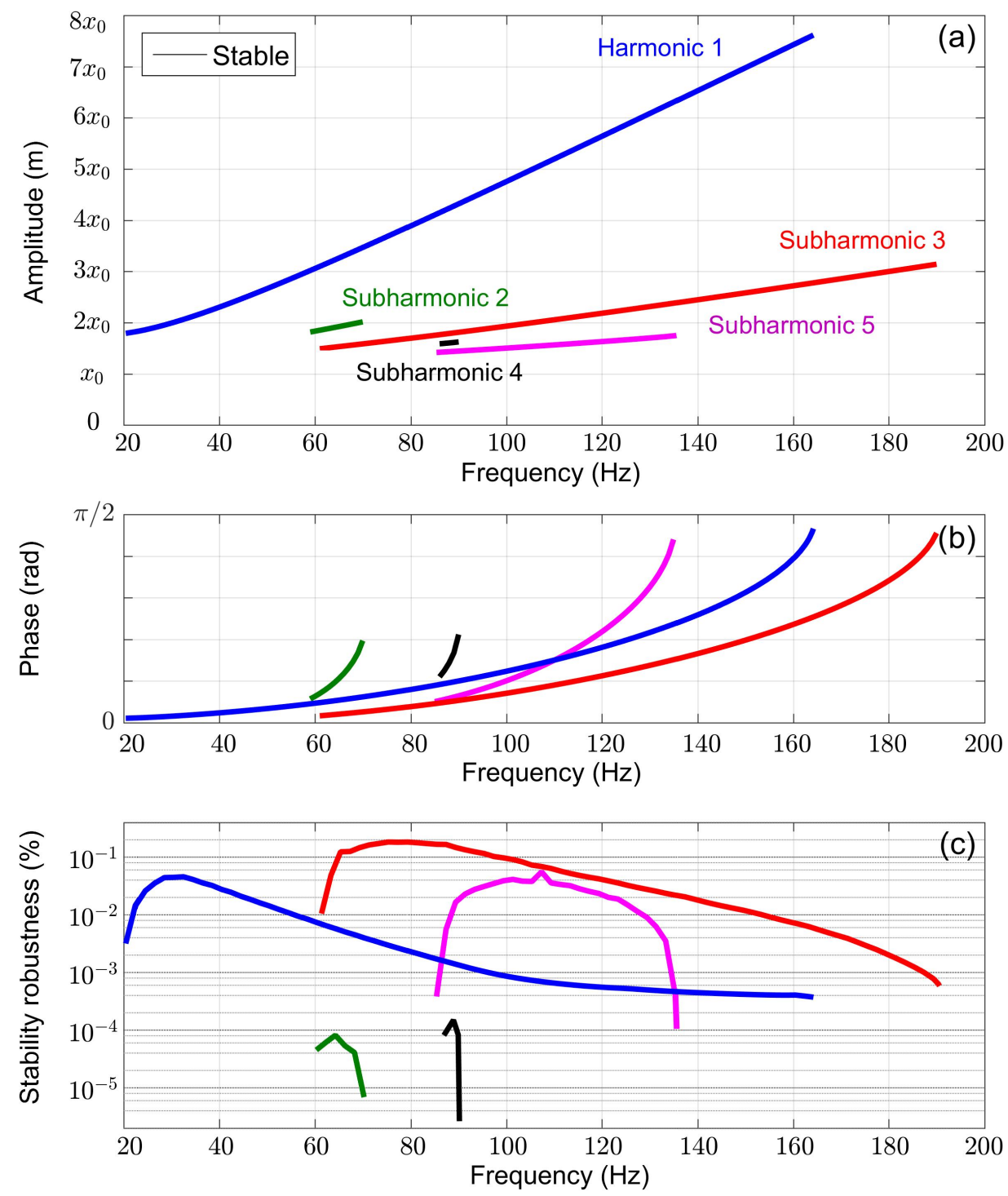

Figure 4: Bistable harvester analytical spectra showing stable high orbits for an excitation amplitude of $5 \mathrm{~m} / \mathrm{s}^{2}$ including the new criterion of stability robustness indicating their sensitivity to disturbances: (a) amplitude, (b) phase of the excitation displacement when the position of the mass reaches a maximum and (c) stability robustness. 
subharmonic ones. Hence, reaching and maintaining even subharmonic behaviors over time will be more complicated than odd subharmonic behaviors, which make them less interesting for energy harvesting purpose. In the same way, the stability robustness of odd subharmonic behaviors is not constant on their whole frequency range. Globally, the robustness decreases as the working point approach the cutting frequency (for a phase of the excitation displacement equal to $\pi / 2$ when the position of the mass reaches a maximum). Harne et al. [17] also reported a similar shape for the robustness of harmonic 1 high orbit with a different approach to calculate the robustness (adding more or less excitation noise to the orbit). The total frequency ranges found for odd subharmonic stable orbits might therefore not be robust enough for energy harvesting. More importantly, it can be seen that harmonic 1 is much less robust than other considered odd harmonics on a wide frequency band, especially in region where it magnitude is high. Therefore, the associated maximum power will not be easily reached (and may actually not be reached at all in real applications). This result also highlights the actual interest of subharmonic behavior (especially subharmonic 3).

From the stability criterion, a threshold can then be defined for the stable high orbit' robustness to only keep the behaviors robust enough for energy harvesting on a long time period.

\section{Experimental analysis}

The previous developments and analyses pointed out the importance of stability robustness for evaluating the true energy harvesting capabilities of nonlinear bistable harvesters, as well as the impact of this criterion on the actual interest of subharmonic behaviors. In order to validate these findings, the present section proposes to experimentally evaluate the performance of an electromagnetic bistable harvester.

\section{V.1. Experimental setup and identification}

Figure 5 shows the experimental setup. The bistable oscillator prototype is made of a brass mass and 2 steel thin beams. The brass mass, itself composed by 3 brass cubes, holds the steel beams in parallel. Those beams are then linked to 2 aluminum supports, situated on both side ends. One support is embedded to the main frame and one is able to translate in the beam direction. A micrometer screw linked to the main frame allows to push this translating supports and to buckle the beams thus creating bistability. The translating support is then fixed during experiment. For symmetry reasons, 2 magnets have been placed on each side of the mass. One of them, coupled to a coil connected to a resistance, enables energy conversion. For optimized energy conversion, the resistance value has been set to be equal to

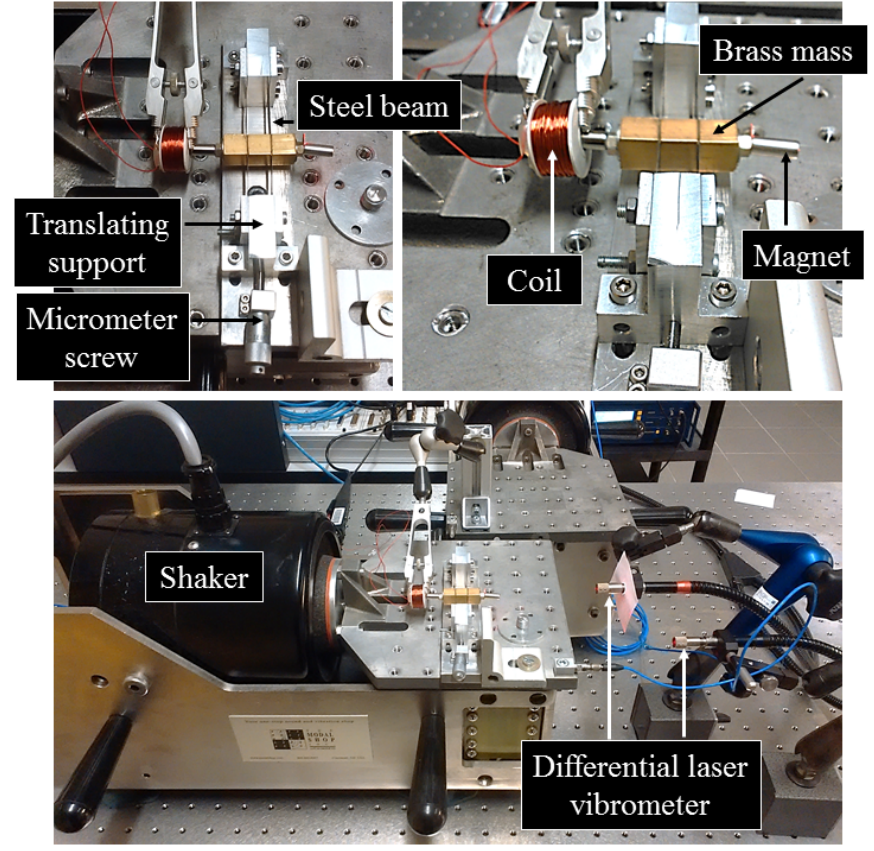

Figure 5: Bistable harvester prototype and experimental setup.

Table 2: Bistable generator prototype dimensions.

\begin{tabular}{lcc}
\hline \hline Element & Value & Unit \\
\hline Inertial mass & & \\
(3 brass cubes and 2 magnets) & 30 & $\mathrm{~g}$ \\
Brass cube dimensions (each) & $10 \times 10 \times 10$ & $\mathrm{~mm}^{3}$ \\
Steel beam dimensions (each) & $28 \times 10 \times 0.1$ & $\mathrm{~mm}^{3}$ \\
Buckling distance reduction & 1.5 & $\mu \mathrm{m}$ \\
Mass stable position & \pm 0.29 & $\mathrm{~mm}$ \\
Coil internal resistance & 18 & $\Omega$ \\
\hline \hline
\end{tabular}

the coil internal resistance (impedance matching). For a given excitation acceleration and frequency, an orbit jump phenomenon can also be obtained thanks to this electromagnetic transducer, through the creation of a force pulse on the bistable oscillator's mass by applying a voltage pulse on the coil. The dimensions of all the elements are presented in Table 2. The buckling coefficient of the bistable oscillator is defined by the ratio between the stable position $x_{0}$ and the beams length. The chosen value of this coefficient in this experimental analysis is quite low ( $1 \%$ ) in order to allow high orbits for acceleration accessible during the experiment (here 5 $\mathrm{m} . \mathrm{s}^{-2}$ ) and representing typical application conditions. This low buckling coefficient also confirms the validity of the Duffing-type mechanical equation to predict the bistable oscillator behaviors.

The bistable harvester prototype is attached to an electrodynamic shaker driven through a feedback loop implemented in a real time controller (DSPace) to impose a constant amplitude sinusoidal acceleration excitation. A differential laser vibrometer measures the relative displacement of the mass with respect to the frame. The following process is then applied to explore the bistable harvester's spectra: (1) the excitation amplitude acceleration is set-up for the entire experience; (2) an excitation 
frequency is chosen; (3) rectangular voltage pulses of $5 \mathrm{~ms}$ of different amplitudes (10 to $50 \mathrm{~V})$ and with various phases are sent to the coil possibly creating orbit jump phenomena; (4) once the orbit under study is reached, the excitation frequency is changed (up or down) slowly and smoothly (the acceleration and its derivatives are kept continuous when changing the frequency) to prevent another orbit jump phenomena; a change in the behavior during this step means that the studied orbit does not exist at this new frequency, or that the behavior is not robust enough to be maintained over time; (5) if the bistable harvester behavior is kept after the frequency change, the average harvested power is calculated from the voltage measured at the terminals of the resistance connected to the coil $\left(P=v_{\text {rms }}^{2} / R\right)$.

The parameters of the bistable harvester mathematical model summarized in Equation (2) are measured for this prototype. Particularly, for small displacements around an equilibrium position, Equation (2) becomes:

$$
\Delta \ddot{x}+\omega_{0}^{2} \Delta x+\left(\frac{\omega_{0}}{Q}+\frac{\beta^{2}}{M\left(R+r_{L}\right)}\right) \Delta \dot{x}=-A \cos (\omega t)
$$

With

$$
\left\{\begin{array}{l}
x=x_{0}+\Delta x \\
\Delta x<<x_{0}
\end{array}\right.
$$

Hence, under such conditions and as reported by Liu et al. [20], the bistable harvester behaves like a linear harvester around its stable positions. In this particular condition, $\omega_{0}$ is the natural frequency of the system which can be determined by measuring its response to a small sharp shock. Two parameters remain to be determined: the quality factor and the stability robustness threshold under which orbits will be considered as non-suitable for energy harvesting. The identification of those 2 parameters need 2 measurements. The quality factor mostly influences the phase of the excitation displacement when the position of the mass reaches a maximum. The stability robustness threshold mostly influence the frequency range limits of the behaviors (acting on the observable cutting frequency). The upper limit of the frequency range of subharmonic 3 behavior has been chosen to identify those 2 parameters. The quality factor has been set to match the phase of the excitation displacement when the position of the mass reaches a maximum for this particular behavior. Similarly, the stability robustness threshold has been set to match the frequency limit of this subharmonic. All the parameters of the bistable prototype are summarized in Table 1.

\section{V.2. Results and discussion}

Experimental results are depicted in Figure 6 and compared to the analytical model predictions for stable high orbits. Figure 6(a)-(b)-(c) respectively represent their amplitude, phase of the excitation displacement when the position of the mass reaches a maximum and stability robustness. For the analytical curves of those figures, a separation is made between orbits robust enough and not robust enough for energy harvesting based on the stability robustness analysis. Analytical high orbits which have a stability robustness under the threshold are considered as non-suitable because of their high sensitivity to external disturbances. Those orbits are thus difficult to reach and maintain over time. Figure 6(d) presents the average harvested power corresponding to the stable high orbits which are robust enough for energy harvesting purpose.

Three different high orbits have been considered, reached and could be maintained over time during the experience: harmonic 1 , subharmonic 3 , and subharmonic 5 orbits. The frequency ranges found for these orbits are respectively $20-60 \mathrm{~Hz}, 65-165 \mathrm{~Hz}$ and $91-130 \mathrm{~Hz}$. The phase of the excitation displacement when the position of the mass reaches a maximum increases with the frequency to respectively $\pi / 25, \pi / 4$ and $\pi / 4$ at the experimental cutting frequency.

Experimental results and analytical predictions are in good agreement, which confirms the pertinence of the Duffing-type model and the pertinence of the novel criterion of stability robustness introduced in this article. The proposed stability robustness evaluation can indeed be used to evaluate the performance of bistable energy harvesters, while a more classical stability analysis may lead to an overestimation of the working ranges and amplitudes. Indeed, the experimental measurements of the phase of the excitation displacement when the position of the mass reaches a maximum show that the theoretical cutting frequency predicted by the classical stability analysis (happening at $\pi / 2$ ) is not reached. The harmonic 1, subharmonic 3 and subharmonic 5 orbits frequency ranges respectively end at $60 \mathrm{~Hz}, 165 \mathrm{~Hz}$ and $130 \mathrm{~Hz}$ for phases of $\pi / 25, \pi / 4$ and $\pi / 4$. Hence, this observation confirms that the common small disturbances stability study is not sufficient to analytically describe the bistable harvester, while experimental and analytical results agreement is obtained once the new notion of stability robustness is included in the analysis. The stability robustness is even more critical for harmonic 1 , as the limit of the high orbits yields an absolute phase that is much smaller than $\pi / 2$, which could be explained by the large displacement obtained when using this behavior.

The even subharmonic orbits are confirmed to be not robust enough to be reached and maintained over time (those orbits have not been found experimentally) and are then logically considered as non-suitable for energy harvesting. Subharmonic 5 orbit presents low average harvested power (maximum of $30 \mu \mathrm{W}$ at $130 \mathrm{~Hz}$ ) compared to harmonic 1 and subharmonic 3 orbits and does not involve any benefit on the bandwidth in the considered example.

A few more particular remarks can then be done that confirm preliminary conclusions drawn from the theoretical analysis. First, it can be noted that the harmonic 1 

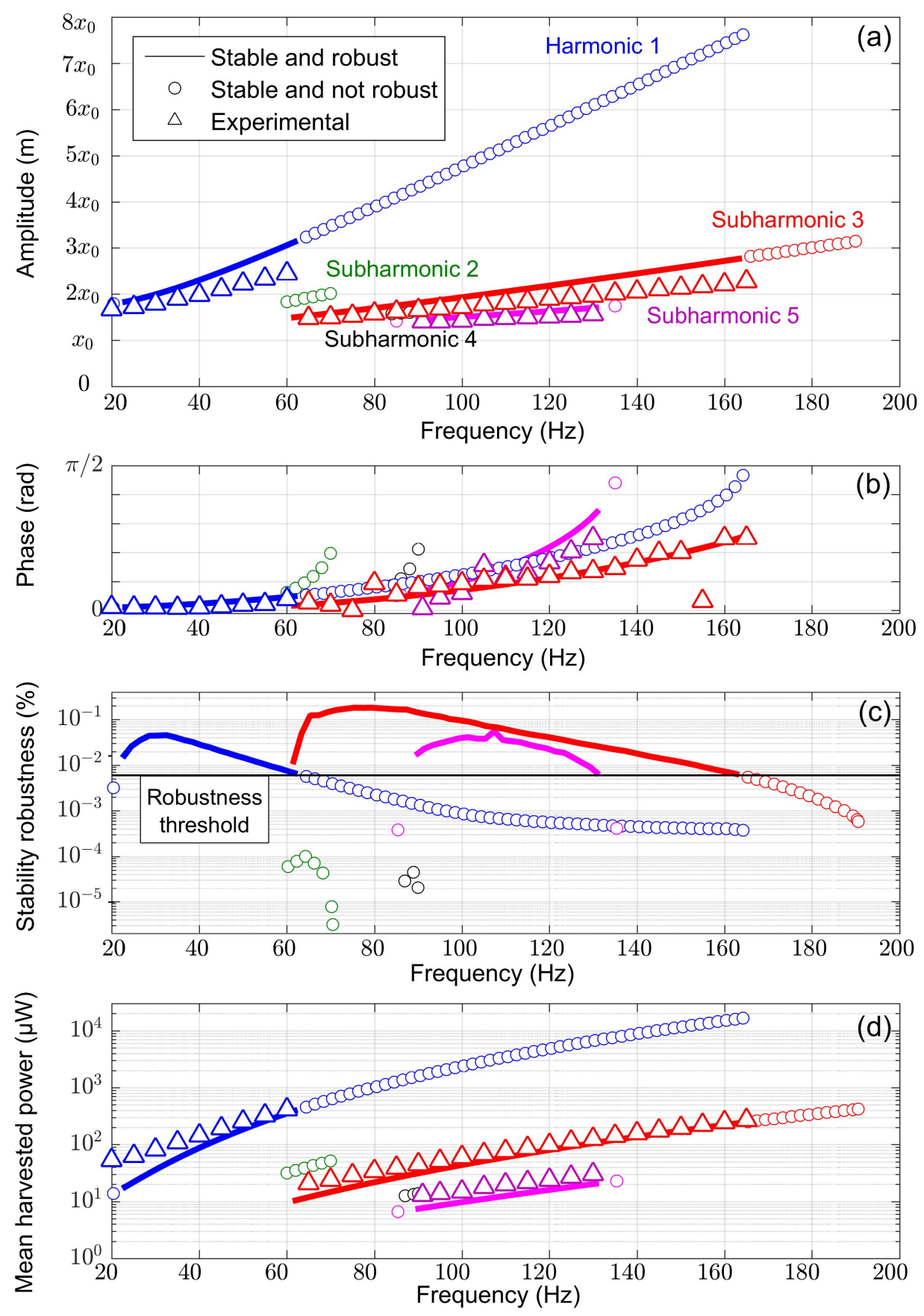

Figure 6: Bistable harvester experimental and analytical spectra showing stable high orbits for an excitation amplitude of $5 \mathrm{~m} / \mathrm{s}^{2}$ including the new criterion of stability robustness indicating their sensitivity to disturbances: (a) amplitude, (b) phase of the excitation displacement when the position of the mass reaches $a$ maximum, (c) stability robustness and $(d)$ average harvested power.

behavior is less interesting than what a classic small disturbances stability study suggests. Its total frequency range is here almost divided by $3(20-160 \mathrm{~Hz}$ with the classical stability analysis and $20-70 \mathrm{~Hz}$ with the stability robustness analysis). On the opposite, the subharmonic 3 behavior is more robust than harmonic 1 behavior and its frequency range is less affected $(60-190 \mathrm{~Hz}$ with the classical stability analysis and $60-160 \mathrm{~Hz}$ with the stability robustness analysis). Moreover, the maximum average power harvested on those behaviors are close to each other $(412 \mu \mathrm{W}$ for harmonic 1 orbit and $269 \mu \mathrm{W}$ for subharmonic 3 orbit).
The efficiency of the prototype have been calculated as the ratio between the ratio between the mean harvested power and the mean power given by the source to the mass. It has been found constant and equal to $10 \%$ on the whole frequency range of both harmonic 1 behavior and subharmonic 3 behavior. Even if this efficiency is under the optimum of $50 \%$ [2], it shows again the pertinence of subharmonic 3 behavior which presents the same efficiency as the harmonic 1 behavior.

Those experimental and analytical results confirm the observations made in the authors' experimental article [15] the subharmonic 3 behavior is an interesting support 
to harmonic 1 behavior to enhance the global operating frequency bandwidth of the bistable harvester for vibrational energy harvesting. Hence, under the experimental conditions, the bistable prototype can harvest more than $100 \mu \mathrm{W}$ on a $70 \mathrm{~Hz}$ frequency band with the combination of these 2 orbits compared to a $25 \mathrm{~Hz}$ frequency band with the sole harmonic 1 orbit, corresponding to a $180 \%$ increase of the useful frequency range.

\section{Conclusion}

A complete analytical analysis has been done for bistable harvesters to predict the different possible steady-state behaviors as a function of the ambient frequency. A particular attention has been put on subharmonic behaviors for which the mass moves $\mathrm{n}$ time slower than the excitation (with $\mathrm{n}$ a natural number) and their ability to support the common harmonic 1 behavior for further enhancing the broadband energy harvesting ability. After applying classical analytical method such as Harmonic Balance method and small disturbances stability analysis, a new criterion has been introduced, namely the stability robustness. Indeed, the stable orbits are not all equivalent being more or less easy to reach and maintain over time. Thus, to include this aspect, the stability robustness indicates the stable orbits sensitivity to disturbances of higher levels. For low stability robustness, the orbits will no longer be considered as suitable for energy harvesting because too sensitive to disturbances for being maintain in time. Finally, an experimental analysis has been conducted to validate the theoretical assumptions.

The experimental results confirm the pertinence of the stability robustness criterion showing good agreement with the analytical predictions. The experimental and predicted behaviors' frequency ranges of the bistable harvester match only once the stability robustness is added to the small disturbances stability analysis. Then, subharmonic 3 behavior appears to be of significant interest for enhancing the global operating frequency range of bistable energy harvesters. It has been found that the bistable prototype harvests more than $100 \mu \mathrm{W}$ on a $70 \mathrm{~Hz}$ frequency band with the combination of harmonic 1 and subharmonic 3 orbits compared to a $25 \mathrm{~Hz}$ frequency band with the sole harmonic 1 orbit, leading to a $180 \%$ increase in the effective bandwidth.

This study having established the analytical analysis of the subharmonic behaviors and their stability robustness in realistic conditions for energy harvesting, future work should focus on the effect of the bistable generator parameters (especially $M, x_{0}, \omega_{0}, R$ ) on those subharmonic behaviors for optimization purposes. Moreover, as some of those behaviors coexist with low orbits which are not interesting for energy harvesting, future work should also focus on techniques to ensure that they will be automatically reached whenever it is possible.

\section{Appendix}

\section{Appendix A}

T-periodic response

$$
x_{m}(t)=a_{m 0}+\sum_{k=1}^{N}\left\{a_{m k} \cos \left(k \frac{\omega}{n} t\right)+b_{m k} \sin \left(k \frac{\omega}{n} t\right)\right\}
$$

Amplitude displacement

$$
\frac{1}{2}\left(\max _{t \in[0, T]}\left(x_{m}(t)\right)-\min _{t \in[0, T]}\left(x_{m}(t)\right)\right)
$$

Phase

$$
\pi-n\left(\tan ^{-1}\left(-b_{m 1} / a_{m 1}\right)+\pi\left(\text { if } a_{m 1}<0\right)\right)
$$

Mean harvested power

$$
\operatorname{mean}_{t \in[0, T]}\left(\frac{\beta^{2}}{R+r_{L}} \dot{x}_{m}^{2}\right)
$$

\section{Appendix B}

$$
\begin{aligned}
\Psi=\sum_{k=1}^{N} & \frac{a_{s k}}{\lambda^{2}+\omega_{k}^{2}}\left(\lambda \cos \left(\omega_{k} t_{0}\right)-\omega_{k} \sin \left(\omega_{k} t_{0}\right)\right) \\
& +\sum_{k=1}^{N} \frac{b_{s k}}{\lambda^{2}+\omega_{k}^{2}}\left(\lambda \sin \left(\omega_{k} t_{0}\right)+\omega_{k} \cos \left(\omega_{k} t_{0}\right)\right)
\end{aligned}
$$

With

$$
\omega_{k}=k \omega / n
$$

\section{Errata}

The following points have been corrected compared to version published in Applied Energy:

- correction in the calculation of the phase. The previous version showed the phase of the mass displacement when the excitation reaches a maximum instead of the phase of the excitation displacement when the position of the mass reaches a maximum. The latter is indeed the most relevant to characterize the bistable behaviors. This phase is generally positive (contrary to the phase shown in the previous version): the frame displacement is ahead of the mass displacement. If this phase is zero, then the displacement of the excitation reaches a maximum when the position of the mass reaches a maximum. If this phase is $\pi$, then the displacement of the excitation reaches a minimum when the position of the mass reaches a maximum. Figures 3, 4 and 6 have been corrected.

- correction of Equation (7): the top right term of the matrix $\underline{\mathrm{M}}$ has been changed from 2 to 1 . 


\section{Acknowledgment}

The authors acknowledge the support of Région Auvergne-Rhône-Alpes through the ARC 4 Energies program.

\section{References}

[1] C. Wei and X. Jing. A comprehensive review on vibration energy harvesting: Modelling and realization. Renewable and Sustainable Energy Reviews, 74:1-18, 2017.

[2] C.B. Williams and R.B. Yates. Analysis Of A Microelectric Generator For Microsystems. Sensors and Actuators A: Physical, 52:8-11, 1996.

[3] A. Erturk, J. Hoffmann, and D.J. Inman. A piezomagnetoelastic structure for broadband vibration energy harvesting. Applied Physics Letters, 94:128130, 2009.

[4] R.L. Harne and K.W. Wang. A review of the recent research on vibration energy harvesting via bistable systems. Smart Materials and Structures, 22:23001, 2013.

[5] H. Vocca, I. Neri, F. Travasso, and L. Gammaitoni. Kinetic energy harvesting with bistable oscillators. Applied Energy, 97:771-776, 2012.

[6] S. Zhou, J. Cao, D.J. Inman, Ji. Lin, S. Liu, and Z. Wang. Broadband tristable energy harvester : Modeling and experiment verification. Applied Energy, 133:33-39, 2014.

[7] D. Younesian and M.R. Alam. Multi-stable mechanisms for high-efficiency and broadband ocean wave energy harvesting. Applied Energy, 197:292302, 2017.

[8] R.L. Harne, M.E. Shoemaker, B.E. Dussault, and K.W. Wang. Wave heave energy conversion using modular multistability. Applied Energy, 130:148-156, 2014.

[9] F.C. Moon and S.W. Shaw. Chaotic vibrations of a beam with non-linear boundary conditions. Nonlinear Mechanics, 18:465-477, 1983.

[10] S.A. Emam and A.H. Nayfeh. Nonlinear responses of buckled beams to subharmonic resonance excitations. Nonlinear Dynamics, 35:105-122, 2004.

[11] A.F. Arrieta, S.A. Neild, and D.J. Wagg. Nonlinear dynamic response and modeling of a bi-stable composite plate for applications to adaptive structures. Nonlinear Dynamics, 58:259-272, 2009.
[12] S.N. Mahmoodi, N. Jalili, and M. Ahmadian. Subharmonics analysis of nonlinear flexural vibrations of piezoelectrically actuated microcantilevers. Nonlinear Dynamics, 59:397-409, 2010.

[13] A.F. Arrieta, P. Hagedorn, A. Erturk, and D.J. Inman. A piezoelectric bistable plate for nonlinear broadband energy harvesting. Applied Physics Letters, 97:104102, 2010.

[14] A. Syta, G. Litak, M.I. Friswell, and S. Adhikari. Multiple solutions and corresponding power output of nonlinear piezoelectric energy harvesters. The European Physical Journal B, 89:99, 2016.

[15] T. Huguet, A. Badel, and M. Lallart. Exploiting bistable oscillator subharmonics for magnified broadband vibration energy harvesting. Applied Physics Letters, 111:173905, 2017.

[16] A.N. Landsbury, J.M. Thompson, and H.B. Stewart. Basin erosion in the twin-well duffing oscillator: two distinct bifurcation scenarios. International Journal of Bifurcation and Chaos, 2:505-532, 1992.

[17] R.L. Harne and Q. Dai. Characterizing the robustness and susceptibility of steady-state dynamics in post-buckled structures to stochastic perturbations. Journal of Sound and Vibration, 395:258-271, 2017.

[18] Theory of Ordinary Differential Equations. Tata McGraw-Hill Education, 1955.

[19] Nonlinear Dispersive Equations: Local and Global Analysis. CBMS Regional Conference Series in Mathematics, 2006.

[20] W.Q. Liu, A. Badel, F. Formosa, Y.P. Wu, and A. Agbossou. Novel piezoelectric bistable oscillator architecture for wideband vibration energy harvesting. Smart Materials and Structures, 22:035013, 2013.

[21] E. Arroyo, A. Badel, F. Formosa, Y.P. Wu, and J. Qiu. Comparison of electromagnetic and piezoelectric vibration energy harvesters: Model and experiments. Sensors and Actuators A: Physical, 183:148-156, 2012.

[22] Micro Energy Harvesting. Wiley, 2015.

[23] G. Floquet. Sur les équations différentielles linéaires à coefficients périodiques. Annales scientifiques de l'ENS, 12:47-88, 1883.

[24] R.L. Harne, M. Thota, and K.W. Wang. Concise and high-fidelity predictive criteria for maximizing performance and robustness of bistable energy harvesters. Applied Physics Letters, 102:053903, 2013. 